Published in final edited form as:

Curr Opin Pediatr. 2016 August ; 28(4): 470-475. doi:10.1097/MOP.0000000000000383.

\title{
Immunogenetics of Cutaneous Lupus Erythematosus
}

\author{
Aimee O. Hersh ${ }^{1}$, Lisa M. Arkin², and Sampath Prahalad ${ }^{3}$ \\ ${ }^{1}$ University of Utah School of Medicine, Salt Lake City, UT \\ ${ }^{2}$ Departments of Dermatology and Pediatrics, University of Wisconsin School of Medicine and \\ Public Health \\ 3Departments of Pediatrics and Human Genetics, Emory University School of Medicine, Atlanta, \\ GA and Children's Healthcare of Atlanta, Atlanta, GA
}

\begin{abstract}
Purpose of the review-Systemic lupus erythematosus (SLE) is the prototypic autoimmune condition, often affecting multiple organ systems, including the skin. Cutaneous lupus (CLE) is distinct from SLE with symptoms isolated to the skin. Histopathologically the hallmark of lupusspecific manifestations of SLE and CLE is an interface dermatitis. The etiology of SLE and CLE is likely multifactorial and may include shared genetic factors. In this review we will discuss the genetic findings related to the cutaneous manifestations of SLE and isolated CLE, with a particular focus on the lupus-specific CLE subtypes.
\end{abstract}

Recent Findings-Several MHC and non-MHC genetic polymorphisms have been identified which may contribute to the cutaneous manifestations of SLE and to CLE. Most of these genetic variants are associated with mechanisms attributed to the pathogenesis of SLE including pathways involved in interferon and vitamin D regulation and UV light exposure. Although there is overlap between the genetic factors associated with SLE and CLE, there appear to be unique genetic factors specific for CLE.

Summary-Improved understanding of the genetics of CLE may lead to the creation of targeted therapies, improving outcomes for patients with this challenging dermatologic condition.

\section{Keywords}

Cutaneous lupus; lupus pathogenesis; genetics

\section{Introduction}

Systemic lupus erythematosus (SLE) is the prototypic autoimmune condition, characterized by the production of autoantibodies leading to organ specific inflammation and damage. SLE can involve multiple organ systems, including the skin. Cutaneous manifestations of SLE are common, affecting up to $75 \%$ of patients with SLE, and may be the first sign of

\footnotetext{
Corresponding author: Aimee Hersh, MD, Pediatric Rheumatology, University of Utah School of Medicine, 81 Mario Capecchi Drive, $4^{\text {th }}$ Floor; Ph: (801) 213-4095, aimee.hersh@ hsc.utah.edu.

Conflicts of Interest: None
} 
disease in 25\% of patients (1). In the 1997 American College of Rheumatology classification criteria for SLE, four of the possible 11 criteria for diagnosing SLE are muco-cutaneous manifestations (malar rash, discoid rash, photosensitivity and nasal/oral ulcerations). The recently updated SLICC classification criteria for SLE diagnosis broadens the potential cutaneous manifestations of SLE to include all subtypes of cutaneous lupus including acute cutaneous lupus (e.g. bullous lupus, the toxic epidermal necrolysis variant of acute cutaneous lupus), subacute cutaneous lupus, chronic cutaneous lupus (e.g. discoid lupus, lupus panniculitis, lupus erythematosus tumidus, chilblain lupus), nasal/oral ulcerations and non-scarring alopecia (2).

Some patients have isolated cutaneous lupus (CLE), which is distinct from SLE. The incidence of CLE is 4.3 per 100,000, which is comparable to the incidence of SLE (3). Not all patients with isolated CLE progress to develop SLE, but population-based studies have suggested a frequency of evolution ranging from $0-28 \%$, with an interval between onset of cutaneous disease and SLE diagnosis ranging from months to 30+ years (3-5). One population-based study demonstrated a sustained risk of systemic disease over time, suggesting that patients should be screened regularly for life (3). Retrospective studies in children suggest a rate of progression to SLE that approaches 30\%, with limited duration of follow up (6).

Cutaneous lupus is typically divided into lupus-specific and lupus-nonspecific manifestations, with lupus-specific disease demonstrating distinct histopathology for lupus including vacuolar interface dermatitis (see Figure 1). Lupus-nonspecific skin lesions are not histopathologically distinct for lupus and may be seen as a reactive feature of another disease process (see Table 1). When present in patients with SLE, lupus non-specific skin disease is often a marker for active systemic disease $(7,8)$.

Lupus-specific disease encompasses 4 different subtypes: acute CLE (ACLE), subacute CLE (SCLE), chronic CLE (CCLE) and a recently defined entity, intermittent CLE (ICLE), which now includes lupus erythematosus tumidus. These subtypes are primarily distinguished by clinical morphology and histologic findings (9). Skin biopsy is often helpful as the many variants of cutaneous lupus can mimic other diseases, and recognition of the specific subset may help to prognosticate risk of developing SLE. A validated scoring system called the Cutaneous Lupus Erythematosus Disease Area and Severity Index (CLASI) was created as a skin-specific outcome instrument to score disease activity and damage in three specific subtypes of CLE (ACLE, SCLE and DLE) (10). Clinical trials in CLE are still uncommon but use of precise outcome instruments may help to determine organ-specific cutaneous response to therapy.

CLE can lead to scarring and dyspigmentation in cosmetically sensitive areas including the face and scalp. Isolated CLE is associated with psychological stress, occupational disability, and severely impaired quality of life. One study demonstrated that patients with CLE experience a poorer quality of life than other common dermatologic conditions including acne, alopecia and skin cancer, and shoulder a mental health burden that matches or exceeds those with chronic hypertension, type 2 diabetes mellitus, recent myocardial infarction, and 
congestive heart failure (11). Importantly, patients with CLE who demonstrate response to treatment through improved skin disease activity scores, have improved quality of life (12).

Finally, disease activity, and not markers of damage including scarring and dyspigmentation, correlates most strongly with impaired quality of life, suggesting that early therapeutic intervention may significantly impact psychological burden in this disease (13).

The etiology of SLE and CLE is likely multifactorial and has been attributed to an interaction between multiple genetic and environmental factors (14). Over the past decade there has been significant interest in understanding the genetic underpinnings of SLE and other related autoimmune conditions. The higher incidence of SLE among family members, and particularly between monozygotic twins, would suggest that there is a genetic predisposition to SLE (15). Numerous studies have demonstrated the increased frequency of certain major histocompatibility complex (MHC) class I and II alleles (e.g. HLA-DR2, $H L A-D R 3$ ), single nucleotide polymorphisms (SNPs) in genes that predispose to multiple autoimmune disorders (e.g. PTPN22, STAT4) as well as variants in genes that are specific to SLE (e.g. ITGAM), although ethnic and population differences may influence these observations (16). Several studies have examined the phenotype/genotype relationship between various SLE disease manifestations and underlying genetic differences. In this manuscript we will review genetic findings related to the cutaneous manifestations of SLE and isolated CLE, with a particular focus on the lupus-specific CLE subtypes.

\section{Genetics of Cutaneous Manifestations of SLE}

A handful of studies have examined the genotype/phenotype relationship between specific SLE manifestations and genotype. The current literature suggests that ITGAM polymorphisms are the most common variants associated with skin involvement in SLE (17). The rs1143679 SNP of ITGAM has been associated with malar rash (ACLE) and discoid lupus (18). ITGAM encodes the $\mathrm{CD} 11 \mathrm{~b}$ chain of the Mac-1 integrin, a surface receptor protein involved in the interaction of monocytes, macrophages and granulocytes. There are several hypotheses as to the role of ITGAM in the pathogenesis of SLE and CLE. One hypothesis is that the production of dysfunctional integrin may prevent cell adhesion and phagocytosis which leads to decreased clearance of extracellular components and increased immune system activation. Specific to the relationship between ITGAM variants and cutaneous SLE, there also appears to be link between ITGAM and photosensitivity with $I T G A M$ variants altering the response to ultraviolet (UV) B irradiation. Lastly, the absence of CD11b may lead to the differentiation of naïve T cells to interleukin-17 (IL-17) producing Th17 cells; increased IL-17 levels have been found in the skin lesions of patients with CLE (19). ITGAM mutations have been associated with several other common manifestations of SLE including renal disease, arthritis and anti-doublestranded DNA, SSA/SSB, RNP and Smith positivity (20).

Sanchez et al described an association between a SNP in the FCGR2A gene (rs1801274) and malar rash in an ethnically heterogeneous cohort of over 8000 SLE patients (OR 1.11, CI 1.17-1.33) (20). FCGR2A encodes an Fc receptor for immunoglobulin $\mathrm{G}$ that is involved in the clearance of immune complexes. The SNP results in an amino acid change that in turn 
results in the generation of an $\mathrm{Fc}$ gamma receptor with altered binding to immunoglobulin $\mathrm{G}$ subclasses.

Variants in the vitamin D receptor ( $V D R)$ gene have also been implicated in susceptibility to cutaneous manifestations of SLE. Vitamin D appears to play a critical role in the prevention of UV-induced DNA damage; photosensitivity and the eruption of erythematous rashes is a well described phenomenon in SLE patients. In a Brazilian population of SLE patients the rs11168268 VDR mutation was associated with "cutaneous alterations" (21). In this same Brazilian population the rs 7805969 polymorphism in the $S T K 17 A$ gene, a DNA repair related gene, was associated with cutaneous manifestations of SLE (22).

Interleukin-6 (IL-6) is a pro-inflammatory cytokine which plays a potential pathogenic role in many inflammatory disorders. Prior studies on the relationship between IL 6 polymorphisms and SLE were inconclusive. To address this, Cui et al performed a metaanalysis of fifteen association studies, and also performed ethnicity-specific meta-analysis in European, Iranian and Asian populations. They found a significant association between a polymorphism in the promoter region of the IL6 gene (-174 G/C SNP; rs1800795) and discoid skin lesions (OR 2.27, 95\% CI 1.05-4.9), supporting a role for this variant in susceptibility to cutaneous manifestations of SLE (23).

\section{Genetics of Cutaneous Lupus Erythematosus}

\section{MHC Associations}

The relationship between chronic cutaneous lupus erythematosus (CCLE) and HLA class II alleles, specifically HLA DQA1, were initially described by Fisher et al in 1994 (24) in a small cohort of 26 patients with CCLE compared with healthy controls. The presence of $D Q A 1 * 0102$ in patients was associated with a 4.6 fold increase of CCLE, which was statistically significant after correcting for multiple comparisons. $H L A D R B 1^{*} 16$ is in linkage disequilibrium with $H L A-D Q A 1^{*} 0102$, and was also observed at an increased frequency, but this association was not significant after correction for multiple comparisons. In another study of 85 Caucasian patients with annular SCLE and DLE, Millard et all found a higher frequency of $\mathrm{H} L A A^{*} 01, B^{*} 08, D R B 1 * 0301$ alleles in patients compared to controls, as well as a significant association between the $H L A A^{*} 03, B^{*} 07$, and the $D R B 1 * 15$ haplotypes and DLE (25). This study also investigated for symptoms of polymorphic light eruption (PLE), an inherited disorder of photosensitivity in patients, their relatives and healthy controls. An association between PLE and cutaneous LE was found ( $\mathrm{p}$ $<0.001$ ), independent of $H L A$ association. They estimated that relative risk of SCLE given the presence of PLE, $D R B 1 * 0301$ and both was 3.37, 5.45 and 12.03 respectively, demonstrating that there may be shared susceptibility to photosensitivity and cutaneous LE.

A recent genome-wide association study identified four novel susceptibility loci for CLE (26). In this study 183 CLE cases (CCLE 44.8\%, SCLE 40.4\%, ICLE (LE tumidus) 14.2\%) and 1288 controls of Central European ancestry were genotyped for over 90,000 SNPs. Replication was performed in 219 subjects and 262 controls from a Finnish cohort. The 4 SNPs that reached genome wide significance (rs2187668, rs9267531, rs4410767 and rs2187668) were all located within the MHC region of chromosome 6. These SNPs were all 
located close to genes involved in various aspects of immune system functioning including antigen presentation, apoptosis regulation, DNA processing and the interferon response (DQA1, MICA, MICB, MSH5, TRIM39 and RPP21).

\section{Non-MHC Associations}

Complement Deficiency_An association between complement deficiency and photosensitive rashes was initially described in 1979 by Levy et al in a 59 year old patient with autosomal recessive inheritance of C2 deficiency (27). Discoid LE has been demonstrated in individual and familial cases of C4 deficiency $(28,29)$. More recently Racila et al described the association between SCLE and a homozygous SNP of the gene $C 1 Q A$, which encodes complement component 1 , subcomponent q, resulting in decreased levels of CIQ (30). Finally, combined partial deficiencies of C2 and C4 were reported in a cohort of adult French patients with CCLE and SCLE in the absence SLE. As most of these patients were smokers, tobacco exposure was hypothesized to be a risk factor for precipitation of their cutaneous disease (31).

TREX 1 Mutations-Mutations in the TREX1 gene are associated with a rare form of cutaneous lupus called familial chilblain lupus (32). Patients with this condition develop painful violaceous nodules, typically on the fingers and toes, that may ulcerate. Patients often have systemic symptoms including neurologic symptoms, as seen in Aicardi Gutierres Syndrome, another syndrome related to dysfunction in the TREX1 gene (33). TREX1 is an intracellular DNAase; deficiency in TREX1 leads to accumulation of components of intracellular DNA which are thought to activate the immune system and lead to autoimmunity.

As with SLE, the ITGAM rs1143679 variant has been associated with isolated CLE, specifically discoid lupus erythematosus (DLE), which is a subtype of CCLE. In a cohort of Finnish and Swedish patients with DLE without signs of systemic disease, there was a significant association with the ITGAM rs1143679 variant (OR 3.2 (95\% CI 2.23-4.57)) (34).

Not surprisingly, other genes implicated in the pathogenesis of SLE have also shown significance in patients with isolated CLE. In a Finnish population with DLE or SCLE the tyrosine kinase 2 (TYK2) rs2304256 SNP was associated with an increased risk of DLE ( $\mathrm{p}=$ $0.012, \mathrm{OR}=1.47,95 \% \mathrm{CI}=1.01-1.98)(35)$. The tyrosine kinase receptor plays a role in interferon signaling; interferon appears to be critical in the pathogenesis of SLE (36). In the same cohort the interferon regulatory factor 5 (IRF5) polymorphism rs 10954213 was associated with DLE ( $\mathrm{p}-0.017, \mathrm{OR}=1.4,95 \% \mathrm{CI}=1.06-1.86)$ and SCLE ( $\mathrm{p}=0.022$, OR $=1.87,95 \%$ CI 1.09-3.21). The IRF5 gene encodes a group of transcription factors which are involved in virus-mediated activation of interferon, modulation of cell growth, apoptosis and immune system activation. A haplotype of cytotoxic T-lymphocyte associated protein 4 (CTLA4) showed association with DLE ( $\mathrm{p}=0.0064, \mathrm{OR}=2.51,95 \% \mathrm{CI}-1.25-5.04)$. CTLA4 is a protein coding gene that transmits an inhibitory signal to T cells. CTLA4 polymorphisms have been associated with multiple autoimmune conditions including SLE, 
Type I diabetes mellitus, Graves disease, Hashimoto thyroiditis and celiac disease and CTLA-4 deficiency may lead to significant immune dysregulation (37).

\title{
Conclusions
}

Several genetic polymorphisms have been identified which may contribute to the cutaneous manifestations of SLE and to CLE. Most of these genetic variants are involved in pathways well described in the pathogenesis of SLE including the roles of interferon, vitamin D regulation and UV light exposure. Although there is overlap between the genetic factors associated with SLE and CLE, there appear to be unique genetic factors specific for CLE. Better understanding of the genetics of CLE may lead to the creation of targeted therapies, improving outcomes for patients with this challenging dermatologic condition.

\section{Acknowledgments}

\begin{abstract}
None
Financial Support and Sponsorship: Research reported in this publication was supported by the National Institute of Arthritis and Musculoskeletal and Skin Diseases of the National Institutes of Health under award number K23AR066064 to Dr. Hersh and by a grant from the Marcus Foundation Inc. to Dr. Prahalad. The content is solely the responsibility of the authors and does not necessarily represent the official views of the National Institutes of Health.
\end{abstract}

\section{References}

1. Tebbe B, Orfanos CE. Epidemiology and socioeconomic impact of skin disease in lupus erythematosus. Lupus. 1997; 6(2):96-104. [PubMed: 9061657]

2. Petri M, Orbai AM, Alarcon GS, Gordon C, Merrill JT, Fortin PR, et al. Derivation and validation of the Systemic Lupus International Collaborating Clinics classification criteria for systemic lupus erythematosus. Arthritis Rheum. 2012; 64(8):2677-2686. [PubMed: 22553077]

3. Durosaro O, Davis MD, Reed KB, Rohlinger AL. Incidence of cutaneous lupus erythematosus, 1965-2005: a population-based study. Arch Dermatol. 2009; 145(3):249-253. [PubMed: 19289752]

4. Gronhagen CM, Fored CM, Granath F, Nyberg F. Cutaneous lupus erythematosus and the association with systemic lupus erythematosus: a population-based cohort of 1088 patients in Sweden. Br J Dermatol. 2011; 164(6):1335-1341. [PubMed: 21574972]

5. Wieczorek IT, Propert KJ, Okawa J, Werth VP. Systemic symptoms in the progression of cutaneous to systemic lupus erythematosus. JAMA Dermatol. 2014; 150(3):291-296. [PubMed: 24477339] This prospective longitudinal cohort study of adults with cutaneous lupus (CLE) found that $17 \%$ of patients progressed to meet ACR criteria for systemic lupus erythematosus (SLE), with a mean time to CLE diagnosis of SLE of eight years. Most met ACR criteria with muco-cutaneous limited disease, and the majority developed none to mild systemic disease during the study period based on SLEDAI criteria. This study suggests that a small percentage of adults with CLE eventually develop SLE, that when they do, most patients will have mild systemic disease.

6. Arkin LM, Ansell L, Rademaker A, Curran ML, Miller ML, Wagner A, et al. The natural history of pediatric-onset discoid lupus erythematosus. J Am Acad Dermatol. 2015; 72(4):628-633. [PubMed: 25648823] This retrospective cohort study of pediatric patients with discoid lupus erythematosus assessed the clinical features and risk of progression to SLE. Nearly $30 \%$ of children who presented with limited-cutaneous disease eventually progressed to meet American College of Rheumatology (ACR) classification criteria for SLE, with greatest risk for SLE diagnosis in the first year after cutaneous diagnosis. Nearly all of those diagnosed with SLE met classification with mucocutaneous limited disease, without developing end-organ involvement through study follow up (median five years). All patients with pediatric-onset DLE require careful monitoring for systemic disease, particularly in the first year after diagnosis. 
7. Gilliam JN, Sontheimer RD. Distinctive cutaneous subsets in the spectrum of lupus erythematosus. J Am Acad Dermatol. 1981; 4(4):471-475. [PubMed: 7229150]

8. Sontheimer RD. The lexicon of cutaneous lupus erythematosus--a review and personal perspective on the nomenclature and classification of the cutaneous manifestations of lupus erythematosus. Lupus. 1997; 6(2):84-95. [PubMed: 9061656]

9. Kuhn A, Landmann A. The classification and diagnosis of cutaneous lupus erythematosus. J Autoimmun. 2014; 48-49:14-19.

10. Bonilla-Martinez ZL, Albrecht J, Troxel AB, Taylor L, Okawa J, Dulay S, et al. The cutaneous lupus erythematosus disease area and severity index: a responsive instrument to measure activity and damage in patients with cutaneous lupus erythematosus. Arch Dermatol. 2008; 144(2):173180. [PubMed: 18283174]

11. Klein R, Moghadam-Kia S, Taylor L, Coley C, Okawa J, LoMonico J, et al. Quality of life in cutaneous lupus erythematosus. J Am Acad Dermatol. 2011; 64(5):849-858. [PubMed: 21397983]

12. Chang AY, Ghazi E, Okawa J, Werth VP. Quality of life differences between responders and nonresponders in the treatment of cutaneous lupus erythematosus. JAMA Dermatol. 2013; 149(1): 104-106. [PubMed: 23324773]

13. Verma SM, Okawa J, Propert KJ, Werth VP. The impact of skin damage due to cutaneous lupus on quality of life. Br J Dermatol. 2014; 170(2):315-321. [PubMed: 24111880] Patients with cutaneous lupus experience poor quality of life that correlates with skin disease activity. This is the first study of its kind to evaluate the impact of lupus-related skin damage (using the Cutaneous Lupus Erythematous Disease Area and Severity Index, the CLASI) on skin-specific quality of life (using the Skindex-29) in a cohort of patients with cutaneous lupus, and to investigate for racial and ethnic disparities. Overall there was no significant correlation between CLASI damage scores and Skindex domains. In addition, dyspigmentation and scarring did not have a significant effect on quality of life. In particular, African Americans with cutaneous lupus experienced damage early in their disease course, frequently in conjunction with disease activity.

14. Achtman JC, Werth VP. Pathophysiology of cutaneous lupus erythematosus. Arthritis Res Ther. 2015; 17:182. [PubMed: 26257198] This review discusses candidate genes that increase the risk of developing CLE. Ultraviolet radiation appears to initiate CLE lesion formation by inducing apoptosis, precipitating auto-antigen presentation, and promoting cellular production of specific cytokines. The exact role of auto-antibodies remains unclear in CLE. Finally, innate immunity is crucial to the pathophysiology of CLE and Th1 cells, TH17 cells, cytotoxic T cells, and invariant natural killer $\mathrm{T}$ cells are all speculated to play a role.

15. Kuo CF, Grainge MJ, Valdes AM, See LC, Luo SF, Yu KH, et al. Familial Aggregation of Systemic Lupus Erythematosus and Coaggregation of Autoimmune Diseases in Affected Families. JAMA Intern Med. 2015; 175(9):1518-1526. [PubMed: 26193127]

16. Graham RR, Hom G, Ortmann W, Behrens TW. Review of recent genome-wide association scans in lupus. J Intern Med. 2009; 265(6):680-688. [PubMed: 19493061]

17. Ceccarelli F, Perricone C, Borgiani P, Ciccacci C, Rufini S, Cipriano E, et al. Genetic Factors in Systemic Lupus Erythematosus: Contribution to Disease Phenotype. J Immunol Res. 2015; 2015:745647. [PubMed: 26798662] This mansucript provides a thorough review of the current knowledge regarding the genetic factors associated with specific disesase manifestations in SLE.

18. Kim-Howard X, Maiti AK, Anaya JM, Bruner GR, Brown E, Merrill JT, et al. ITGAM coding variant (rs1143679) influences the risk of renal disease, discoid rash and immunological manifestations in patients with systemic lupus erythematosus with European ancestry. Ann Rheum Dis. 2010; 69(7):1329-1332. [PubMed: 19939855]

19. Oh SH, Roh HJ, Kwon JE, Lee SH, Kim JY, Choi HJ, et al. Expression of interleukin-17 is correlated with interferon-alpha expression in cutaneous lesions of lupus erythematosus. Clin Exp Dermatol. 2011; 36(5):512-520. [PubMed: 21631571]

20. Sanchez E, Nadig A, Richardson BC, Freedman BI, Kaufman KM, Kelly JA, et al. Phenotypic associations of genetic susceptibility loci in systemic lupus erythematosus. Ann Rheum Dis. 2011; 70(10):1752-1757. [PubMed: 21719445]

21. de Azevedo Silva J, Monteiro Fernandes K, Tres Pancotto JA, Sotero Fragoso T, Donadi EA, Crovella $S$, et al. Vitamin D receptor (VDR) gene polymorphisms and susceptibility to systemic 
lupus erythematosus clinical manifestations. Lupus. 2013; 22(11):1110-1117. [PubMed: 23945129]

22. da Silva Fonseca AM, de Azevedo Silva J, Pancotto JA, Donadi EA, Segat L, Crovella S, et al. Polymorphisms in STK17A gene are associated with systemic lupus erythematosus and its clinical manifestations. Gene. 2013; 527(2):435-439. [PubMed: 23860322]

23. Cui YX, Fu CW, Jiang F, Ye LX, Meng W. Association of the interleukin-6 polymorphisms with systemic lupus erythematosus: a meta-analysis. Lupus. 2015; 24(12):1308-1317. [PubMed: 26038346]

24. Fischer GF, Pickl WF, Fae I, Anegg B, Milota S, Volc-Platzer B. Association between chronic cutaneous lupus erythematosus and HLA class II alleles. Hum Immunol. 1994; 41(4):280-284. [PubMed: 7883595]

25. Millard TP, Kondeatis E, Vaughan RW, Lewis CM, Khamashta MA, Hughes GR, et al. Polymorphic light eruption and the HLA DRB $1 * 0301$ extended haplotype are independent risk factors for cutaneous lupus erythematosus. Lupus. 2001; 10(7):473-479. [PubMed: 11480844]

26. Kunz M, Konig IR, Schillert A, Kruppa J, Ziegler A, Grallert H, et al. Genome-wide association study identifies new susceptibility loci for cutaneous lupus erythematosus. Exp Dermatol. 2015; 24(7):510-515. [PubMed: 25827949] This is the first genome-wide assocation study of CLE.

27. Levy SB, Pinnell SR, Meadows L, Snyderman R, Ward FE. Hereditary C2 deficiency associated with cutaneous lupus erythematosus: clinical, laboratory, and genetic studies. Arch Dermatol. 1979; 115(1):57-61. [PubMed: 760659]

28. Agnello V, Gell J, Tye MJ. Partial genetic deficiency of the C4 component of complement in discoid lupus erythematosus and urticaria/angioedema. J Am Acad Dermatol. 1983; 9(6):894-898. [PubMed: 6643787]

29. Voigtlander V, Bahmer F, Hauptmann G. Familial discoid lupus erythematosus associated with heterozygous C4 deficiency. Acta Derm Venereol. 1984; 64(6):552-554. [PubMed: 6084933]

30. Racila DM, Sontheimer CJ, Sheffield A, Wisnieski JJ, Racila E, Sontheimer RD. Homozygous single nucleotide polymorphism of the complement C1QA gene is associated with decreased levels of C1q in patients with subacute cutaneous lupus erythematosus. Lupus. 2003; 12(2):124132. [PubMed: 12630757]

31. Boeckler P, Milea M, Meyer A, Uring-Lambert B, Heid E, Hauptmann G, et al. The combination of complement deficiency and cigarette smoking as risk factor for cutaneous lupus erythematosus in men; a focus on combined C2/C4 deficiency. Br J Dermatol. 2005; 152(2):265-270. [PubMed: 15727637]

32. Rice G, Newman WG, Dean J, Patrick T, Parmar R, Flintoff K, et al. Heterozygous mutations in TREX1 cause familial chilblain lupus and dominant Aicardi-Goutieres syndrome. Am J Hum Genet. 2007; 80(4):811-815. [PubMed: 17357087]

33. Rice G, Patrick T, Parmar R, Taylor CF, Aeby A, Aicardi J, et al. Clinical and molecular phenotype of Aicardi-Goutieres syndrome. Am J Hum Genet. 2007; 81(4):713-725. [PubMed: 17846997]

34. Jarvinen TM, Hellquist A, Koskenmies S, Einarsdottir E, Panelius J, Hasan T, et al. Polymorphisms of the ITGAM gene confer higher risk of discoid cutaneous than of systemic lupus erythematosus. PLoS One. 2010; 5(12):e14212. [PubMed: 21151989]

35. Jarvinen TM, Hellquist A, Koskenmies S, Einarsdottir E, Koskinen LL, Jeskanen L, et al. Tyrosine kinase 2 and interferon regulatory factor 5 polymorphisms are associated with discoid and subacute cutaneous lupus erythematosus. Exp Dermatol. 2010; 19(2):123-131. [PubMed: 19758313]

36. Ghodke-Puranik Y, Niewold TB. Immunogenetics of systemic lupus erythematosus: A comprehensive review. J Autoimmun. 2015; 64:125-136. [PubMed: 26324017]

37. Kuehn HS, Ouyang W, Lo B, Deenick EK, Niemela JE, Avery DT, et al. Immune dysregulation in human subjects with heterozygous germline mutations in CTLA4. Science. 2014; 345(6204): 1623-1627. [PubMed: 25213377]

38. Kuhn, A.; Lehmann, P.; Ruzicka, T. Cutaneous lupus erythematosus. Berlin: Spring-Verlag; 2004. p. 53-58.

39. Werth VP. Clinical manifestations of cutaneous lupus erythematosus. Autoimmun Rev. 2005; 4(5): 296-302. [PubMed: 15990077] 


\section{Key Phrases}

- $\quad$ Cutaneous involvement is common in systemic lupus erythematosus (SLE).

- $\quad$ There are 4 subtypes of lupus-specific cutaneous lupus (CLE); histopathologically the hallmark of lupus-specific manifestations of SLE and CLE is an interface dermatitis.

- $\quad$ The etiology of SLE and CLE is likely multifactorial but there may a shared genetic susceptibility. 


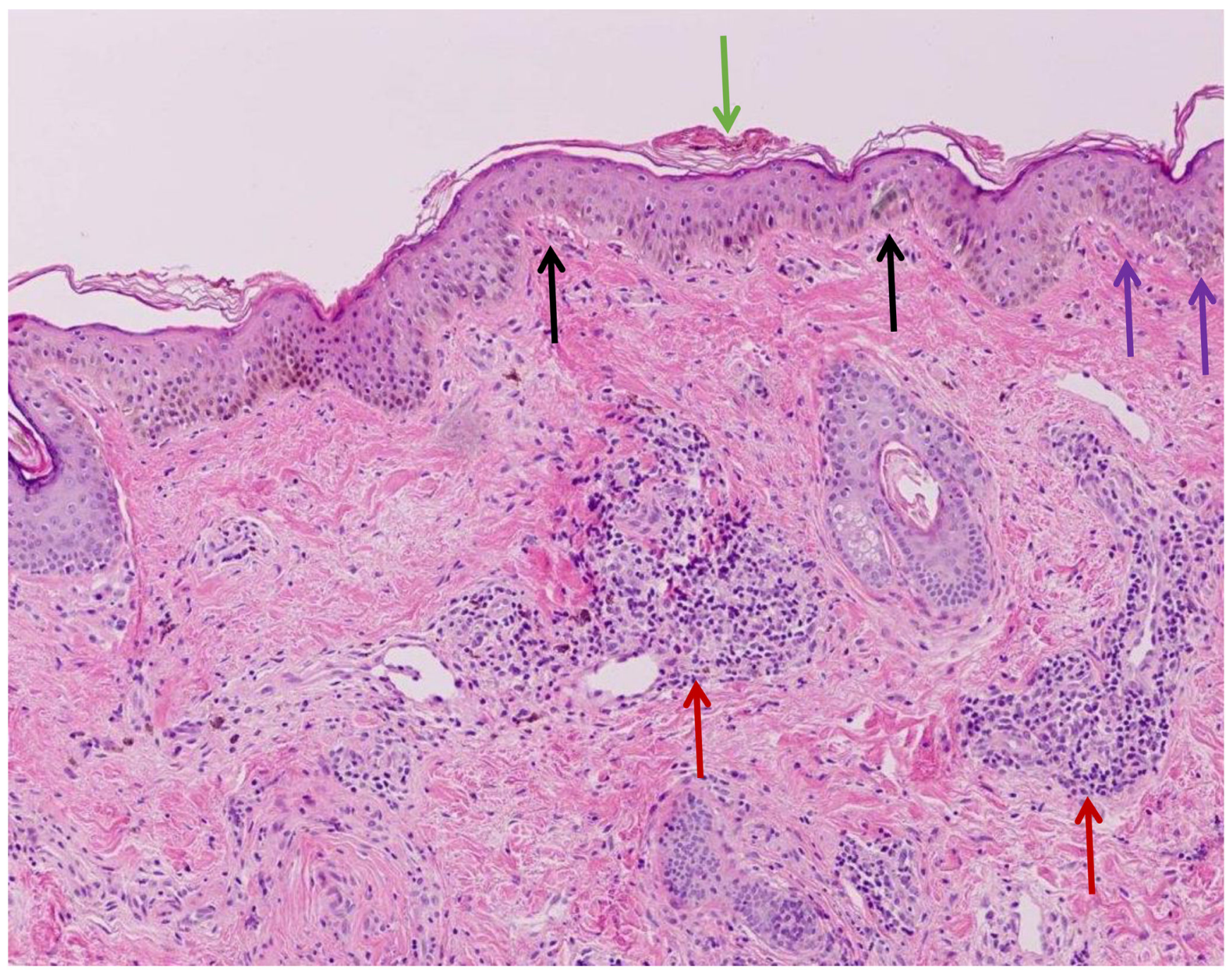

Figure 1.

Classic histopathology for discoid lupus erythematosus is demonstrated including hyperkeratosis of the stratum corneum (green arrow), mild thickening of the basement membrane (purple arrows), and vacuolar interface dermatitis at the dermal-epidermal junction (black arrows). There are also lymphoid infiltrates surrounding both blood vessels and eccrine glands in the dermis (red arrows). 


\section{Table 1}

Lupus-specific and non-specific manifestations, based on the modified Giliam and Dusseldorf Classification for Cutaneous Lupus $(7,38,39)$.

\begin{tabular}{|c|c|c|c|}
\hline \multirow[t]{4}{*}{1} & \multicolumn{3}{|c|}{ Acute Cutaneous Lupus Erythematosus(ACLE) } \\
\hline & a. & \multicolumn{2}{|c|}{ Localized form (malar rash) } \\
\hline & b. & \multicolumn{2}{|c|}{ Generalized form (morbiliform) } \\
\hline & c. & \multicolumn{2}{|c|}{ TEN-like ACLE } \\
\hline \multirow[t]{3}{*}{2} & \multicolumn{3}{|c|}{ Subacute Cutaneous Lupus Erythematosus (SCLE) } \\
\hline & a. & \multicolumn{2}{|c|}{ Annular SCLE } \\
\hline & b. & \multicolumn{2}{|c|}{ Papulosquamous/psoriasiform } \\
\hline \multirow[t]{2}{*}{3} & \multicolumn{3}{|c|}{ Intermittent Cutaneous Lupus Erythematosus (ICLE) } \\
\hline & a. & \multicolumn{2}{|c|}{ Lupus erythematosus tumidus } \\
\hline \multirow[t]{12}{*}{4} & \multicolumn{3}{|c|}{ Chronic Cutaneous Lupus Erythematosus (CCLE) } \\
\hline & a. & \multicolumn{2}{|c|}{ Discoid lupus Erythematosus (DLE) } \\
\hline & & i. & Localized \\
\hline & & ii. & Generalized \\
\hline & \multirow[t]{4}{*}{ b. } & \multicolumn{2}{|c|}{ Mucosal DLE } \\
\hline & & i. & Oral \\
\hline & & ii. & Conjunctival \\
\hline & & iii. & Genital \\
\hline & c. & \multicolumn{2}{|c|}{ Hypertrophic/Verrucous DLE } \\
\hline & d. & \multicolumn{2}{|c|}{ Lupus panniculitis/profundus } \\
\hline & e. & \multicolumn{2}{|c|}{ Chillblain lupus } \\
\hline & f. & \multicolumn{2}{|c|}{ Lichenoid (Lupus Erythematosus/Lichen Planus Overlap) } \\
\hline
\end{tabular}

Lupus non-specific manifstations:

1

Cutaneous vascular disease

a.

Vasculitis:

i. Leukocytoclastic vasculitis

ii. Urticarial vasculitis

b. Vasculopathy:

i. Degos' disease

ii. Secondary atrophie blanche

c. Periungual telangiectasias

d. Livedo reticularis

e. Thrombophlebitis

f. Raynaud's phenomenon

g. Erythromelalgia

$2 \quad$ Non-scarring alopecia
a. Telogen effluvium
b. "Lupus" hair
c. Alopecia areata

Curr Opin Pediatr. Author manuscript; available in PMC 2017 August 01. 


\begin{tabular}{|c|c|c|}
\hline & 3 & Rheumatoid nodules \\
\hline & 4 & Calcinosis cutis \\
\hline$D$ & 5 & LE non-specific bullous lesions \\
\hline$\dot{\vec{\partial}}$ & 6 & Urticaria \\
\hline 은 & 7 & Papulonodular mucinosis \\
\hline 及 & 8 & Anetoderma/cutis laxa \\
\hline$\stackrel{2}{\complement}$ & 9 & Acanthosis nigricans \\
\hline 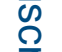 & 10 & Erythema multiforme \\
\hline$\overline{5}$ & 11 & Leg ulcers \\
\hline & 12 & Lichen planus \\
\hline
\end{tabular}

